\title{
Dépôts atmosphériques azotés et leurs effets en forêt: un bilan des sites d'observation à long terme
}

\author{
Anne Thimonier \\ Elisabeth Graf Pannatier \\ Maria Schmitt \\ Peter Waldner \\ Patrick Schleppi \\ Sabine Braun
}

Institut fédéral de recherches sur la forêt, la neige et le paysage $(\mathrm{CH})^{*}$ Institut fédéral de recherches sur la forêt, la neige et le paysage $(\mathrm{CH})$ Institut fédéral de recherches sur la forêt, la neige et le paysage $(\mathrm{CH})$ Institut fédéral de recherches sur la forêt, la neige et le paysage $(\mathrm{CH})$ Institut fédéral de recherches sur la forêt, la neige et le paysage $(\mathrm{CH})$ Institut de biologie végétale appliquée IBA/IAP $(\mathrm{CH})$

\author{
Nitrogen deposition and its effects in forests: results from long-term \\ observation sites?
}

\begin{abstract}
Emissions of nitrogenous pollutants reached a peak in the mid-1980s and have decreased since then, but they remain at a high level. Long-term observation sites were established between 1984 and 1995 within three networks in Switzerland (IAP, LWF and NITREX), following growing concern that the vitality of forests might be affected by air pollution. Here we assess the nitrogen status of selected sites and its changes over the last 15 to 25 years. Nitrogen deposition at the sites was either obtained from models (IAP) or from throughfall measurements (LWF and NITREX). Indicators of the nitrogen status of a site included nitrate leaching from the rooting zone (43 sites), tree foliar concentrations of nitrogen and other nutrients ( $>100$ sites), and the species composition of ground vegetation (59 sites). Nitrogen deposition has significantly decreased on eight out of twelve sites during the last 15 years. However, except in the Alps, deposition remains within or above the range of empirical critical loads, which correspond to the deposition levels above which the ecosystem might be negatively affected. Nitrate leaching can be locally high, depending on deposition rates, but also on other factors such as soil properties. Tree foliar concentrations in nitrogen and phosphorus have tended to decrease during the last 15 to 25 years. The comparison of vegetation surveys, first carried out in 1984, then repeated in 2003/2004, indicated an increase in nitrophilous species (IAP), but on a more recent and shorter period (1994/1998-2011), on a smaller number of sites (LWF), we found no significant changes.
\end{abstract}

Keywords: forest ecosystems, nitrogen deposition, foliar analyses, nitrate leaching, ground vegetation, longterm observation sites

doi: $10.3188 /$ szf.2012.0343

*Zürcherstrasse 111, CH-8903 Birmensdorf, courriel anne.thimonier@wsl.ch

A vec le développement des activités agricoles et industrielles et l'augmentation du parc automobile, les émissions d'azote liées aux activités humaines ont augmenté massivement à partir des années 1950, en Suisse comme dans le reste de l'Europe (CFHA 2005). Au cours des années 1990, des stratégies ont été mises en œuvre afin de réduire les émissions d'azote dans l'atmosphère. Les mesures prises ont permis de réduire efficacement les émissions de composés azotés par les transports, l'industrie et les ménages. Ainsi, les émissions d'oxydes d'azote $\left(\mathrm{NO}_{\mathrm{X}}\right)$, formés essentiellement lors des processus de combustion, ont été pratiquement divisées par deux entre 1994 et 2005 (Heldstab et al 2010). En revanche, les émissions d'azote ammoniacal $\left(\mathrm{NH}_{3}\right)$, provenant essentiellement des activités agricoles, restent élevées.
L'azote émis dans l'atmosphère est transporté à plus ou moins longue distance avant de se déposer sous forme de pluie, gaz ou particules dans les milieux terrestres et aquatiques. L'azote déposé se trouvant sous une forme facilement utilisable par les végétaux, la disponibilité de cet élément s'est accrue considérablement dans des milieux où l'azote était naturellement une ressource limitante, comme dans les écosystèmes forestiers. Ce surplus d'azote est donc à même d'exercer un effet fertilisant sur la croissance des arbres (Solberg et al 2009). Cependant, comme c'est surtout la production de biomasse aérienne (bois et feuillage) qui est stimulée, au détriment de la production de racines (Flückiger \& Braun 1998), l'effet fertilisant de l'azote a le revers de rendre les arbres potentiellement plus sensibles à des événements climatiques comme une sécheresse prolongée. 
De plus, un excès d'azote par rapport aux autres éléments minéraux peut provoquer des déséquilibres nutritifs, qui à leur tour peuvent être à l'origine d'une sensibilité accrue des arbres à des facteurs de stress comme le gel ou les attaques parasitaires (Flückiger \& Braun 1998; Erisman \& de Vries 2000). La disponibilité en azote $(\mathrm{N})$ ayant augmenté, d'autres éléments essentiels, le phosphore $(\mathrm{P})$ notamment, pourraient désormais limiter la croissance des arbres (Braun et al 2010).

Au-delà de son effet fertilisant, l'azote participe également au processus d'acidification des sols (Erisman \& de Vries 2000). Le prélèvement par les racines des arbres d'azote sous forme ammoniacale $\left(\mathrm{NH}_{4}{ }^{+}\right)$et la nitrification (transformation de $\mathrm{NH}_{4}{ }^{+}$en nitrate $\left.\mathrm{NO}_{3}{ }^{-}\right)$conduisent à la libération de protons $\left(\mathrm{H}^{+}\right)$dans l'eau du sol. Si le nitrate est en excès par rapport à la demande des végétaux et des microorganismes du sol pour leur croissance, il sort de l'écosystème par lessivage en dessous de la zone racinaire (Aber et al 1998). En entraînant avec lui des cations nutritifs essentiels comme le calcium, le magnésium et le potassium, le nitrate lessivé contribue à l'acidification et l'appauvrissement du sol. L'écosystème est dit saturé en azote lorsque le lessivage atteint des proportions significatives, de l'ordre de $2-4 \mathrm{~kg} \mathrm{~N} /$ (ha $\times$ an) d'azote dans nos forêts (Slootweg et al 2007). Le lessivage de nitrate est un indicateur de risques accrus pour un écosystème. Il intervient dans l'évaluation des «charges critiques», qui correspondent aux dépôts au-dessous desquels on n'observe pas d'effet négatif sur l'écosystème étudié (Braun et al 2012, ce numéro). Ces charges critiques peuvent être modélisées individuellement pour chaque site par la méthode des bilans de masse à l'équilibre, une méthode qui prend en compte les flux d'entrée et de sortie d'azote dans un écosystème donné. Elles peuvent être également déterminées em-

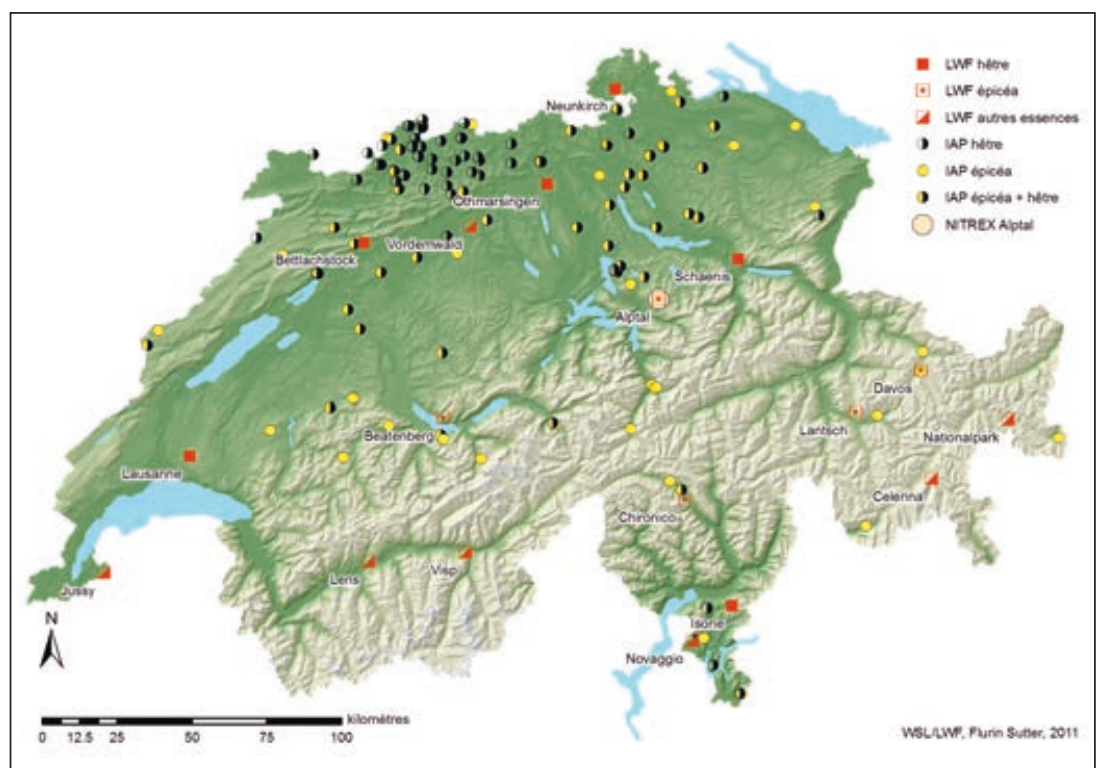

Fig. 1 Localisation des sites d'observation à long terme appartenant aux réseaux IAP, LWF et NITREX. Les différents symboles indiquent l'essence principale rencontrée sur le site. Source: IAP/LWF piriquement sur la base d'observations et d'expérimentations. La gamme de valeurs des charges critiques empiriques ainsi déterminées s'étend, selon la sensibilité de l'écosystème et la disponibilité des données, de 10 à $20 \mathrm{~kg} \mathrm{~N} /($ ha $\times$ an) pour les forêts de feuillus et de 5 à $15 \mathrm{~kg} \mathrm{~N} /($ ha $\times$ an) pour les forêts de conifères (Bobbink \& Hettelingh 2011).

Dans cet article, nous présentons quelques résultats d'études à long terme, menées sur des sites permanents établis dans le cadre de trois réseaux d'observation des écosystèmes forestiers. Notre premier objectif est de présenter, sur une sélection de sites, les dépôts azotés et leur évolution depuis les premières années de mesures au milieu des années 1990, et comparer ces dépôts à l'évolution des émissions d'azote et aux charges critiques. Le second objectif est de caractériser la disponibilité en azote des écosystèmes forestiers au moyen de trois indicateurs, dont nous examinerons l'évolution au cours du temps. Il s'agit du lessivage de nitrate en dessous de la zone d'enracinement, un indicateur de l'état de saturation de l'écosystème à l'égard de l'azote; des concentrations du feuillage des arbres en éléments nutritifs, indicatrices de la qualité de la nutrition du peuplement forestier; et de la composition floristique de la végétation du sous-bois, une composante de l'écosystème qui reflète assez fidèlement les conditions environnementales et qui est susceptible de réagir relativement vite à des modifications de celles-ci.

\section{Matériel et méthodes}

\section{Sites}

Les sites permanents faisant l'objet de cette contribution appartiennent à trois réseaux. Le réseau géré par l'IAP (Institut de biologie végétale appliquée, à Schönenbuch) est le plus ancien. Mandaté par différents cantons suisses ainsi que par l'Office fédéral de l'environnement, l'IAP a mis en place 51 sites permanents en 1984. Au cours des années suivantes, l'IAP a installé un nombre croissant de sites dans les différentes régions de Suisse, sur une large gamme de types de sols, d'altitudes et de niveaux de pollution. Le réseau compte actuellement un total de 135 sites, établis dans des peuplements matures de hêtre, d'épicéa, ou mixtes (figure 1). L'âge moyen des hêtres est de 125 ans (entre 30 et 180), celui des épicéas est de 145 ans (entre 30 et 310).

Le deuxième réseau, établi dans le cadre du programme de recherches à long terme sur les écosystèmes forestiers LWF, a été mis en place en 1994 (Innes 1995). Le réseau LWF fait partie du réseau du programme européen d'observation intensive des forêts «PIC-Forêts» (de Vries et al 2003). Il est géré par l'Institut fédéral de recherches sur la forêt, la neige et le paysage (WSL), basé à Birmensdorf, et il est soutenu financièrement par l'Office fédéral de l'environ- 
nement. Le réseau LWF compte un total de 18 sites (figure 1), répartis dans l'ensemble de la Suisse, dans des peuplements forestiers dont l'essence principale est variable. Parmi les essences les plus représentées figurent le hêtre (6 sites) et l'épicéa (5 sites). L'âge des hêtres varie de 70 à 210 ans, celui des épicéas de 160 à 250 ans.

Le troisième réseau a été établi initialement dans le cadre du projet Européen NITREX («Nitrogen Saturation Experiments»; Wright \& Rasmussen 1998). L'objectif de NITREX était d'étudier le cycle biogéochimique d'éléments dans divers écosystèmes forestiers de conifères, soumis expérimentalement à différents niveaux de dépôts d'azote. Les sites de ce réseau ont été établis dans divers pays d'Europe, parmi lesquels la Suisse. Le site suisse affilié à NITREX a été installé en Suisse centrale dans une forêt subalpine d'épicéa, à Alptal (figure 1). Sur ce site, géré par le WSL, une expérience d'addition d'azote a débuté en 1995 et continue à ce jour. Le site consiste en deux bassins expérimentaux, l'un recevant des apports d'azote simulant des dépôts atmosphériques supplémentaires de $25 \mathrm{~kg} \mathrm{~N} /($ ha $\times$ an), le deuxième servant de témoin et ne recevant qu'un supplément d'eau (Schleppi et al 2006a). Dans cette contribution, nous présenterons quelques résultats provenant du bassin témoin.

\section{Dépôts atmosphériques d'azote}

Les dépôts azotés constituent un des facteurs majeurs pouvant influencer le fonctionnement des écosystèmes forestiers. Une des méthodes permettant de quantifier ces dépôts consiste à échantillonner en parallèle les précipitations à découvert, dans une clairière par exemple, et sous couvert forestier («pluviolessivats»). Les apports d'azote mesurés hors couvert correspondent approximativement aux dépôts sous forme humide, c'est-à-dire sous forme de pluie ou neige. Les pluviolessivats résultent quant à eux des apports sous forme humide et sous forme sèche, car les précipitations mobilisent les substances déposées sur les branches et le feuillage lors de la période précédant une averse. Cependant, les processus d'échange dans le houppier (absorption ou au contraire libération de certains ions) contribuent à modifier la composition des pluviolessivats. Dans le cas de l'azote, une partie des apports atmosphériques est prélevée directement par le feuillage. Différents modèles de bilan au niveau de la canopée permettent de simuler ces processus d'échange et d'estimer ainsi les dépôts atmosphériques totaux, c'est-à-dire la somme des dépôts secs et humides.

Au sein du réseau IAP, les pluviolessivats ont été mesurés sur 30 sites, mais sur des périodes de durée variable et échelonnées dans le temps (Flückiger $\&$ Braun 1998). Afin de travailler sur un jeu de données homogène et de pouvoir inclure l'ensemble des sites dans l'analyse, nous avons utilisé les dépôts modélisés par Rihm (1994).
Sur 13 des 18 sites LWF et à Alptal, les dépôts azotés sont quantifiés par la méthode des pluviolessivats (Thimonier 1998, Thimonier et al 2005). Le modèle de bilan appliqué aux pluviolessivats mesurés à Alptal et sur les sites LWF est détaillé dans Thimonier et al (2005). Ce modèle sous-estime l'absorption directe de l'azote gazeux (ammoniac $\mathrm{NH}_{3}$ et dioxyde d'azote $\mathrm{NO}_{2}$; Mohr et al 2005). Les dépôts totaux d'azote réels sont donc plus élevés que les dépôts estimés par cette méthode.

Sur les sites LWF, des collecteurs, constitués de bouteilles munies d'entonnoirs, recueillent les pluviolessivats. Sur les sites d'altitude, où les précipitations neigeuses peuvent être abondantes, ces collecteurs sont remplacés par des seaux à neige en hiver. Hors couvert, des collecteurs de type entonnoir ou un seau à neige, selon la saison et le site, recueillent les précipitations incidentes. Ces collecteurs sont prélevés toutes les deux semaines en règle générale, afin de déterminer la composition chimique des précipitations en laboratoire. Les collecteurs de précipitation, leur positionnement sur le site et les paramètres analysés en laboratoire sont décrits plus en détail dans Thimonier et al (2005).

Sur le site d'Alptal, les précipitations sont échantillonnées par deux collecteurs de type entonnoir hors couvert, quatre collecteurs de même type sous couvert. En hiver, les collecteurs sous couvert sont remplacés par autant de seaux à neige. Les prélèvements ont lieu toutes les semaines (Schleppi et al 1998).

\section{Lessivage de nitrate}

Sur 40 sites IAP, l'eau du sol est prélevée une fois par mois à l'aide de lysimètres à succion. Les lysimètres sont installés à 20,40 et $80 \mathrm{~cm}$ de profondeur en règle générale (Braun \& Flückiger 2012). Huit bougies en céramique sont installées pour chaque profondeur. Les échantillons d'eau recueillis sont mélangés par profondeur, puis analysés en laboratoire. Afin de quantifier le lessivage de nitrate en dessous de la zone d'enracinement, les concentrations en nitrate mesurées à la profondeur la plus basse (en général $80 \mathrm{~cm}$ ) sont multipliées par un flux d'eau de percolation calculé à l'aide du modèle WASIM-ETH (Schulla \& Jasper 2007).

Sur 7 des 13 sites LWF pour lesquels les pluviolessivats sont échantillonnés, l'eau du sol est prélevée toutes les deux semaines depuis 1999 ou 2000, au même rythme que les précipitations. Elle est collectée en huit points de chaque site à l'aide de plaques gravitaires sous la litière et par succion $(500 \mathrm{hPa})$ à l'aide de lysimètres avec bougies en céramique à 15 , 50 et $80 \mathrm{~cm}$ de profondeur (Graf Pannatier et al 2004, Graf Pannatier et al 2009). Les échantillons recueillis sont ensuite analysés en laboratoire. Pour le calcul du lessivage de nitrate, les concentrations en nitrate mesurées à $80 \mathrm{~cm}$ de profondeur sont multipliées par 


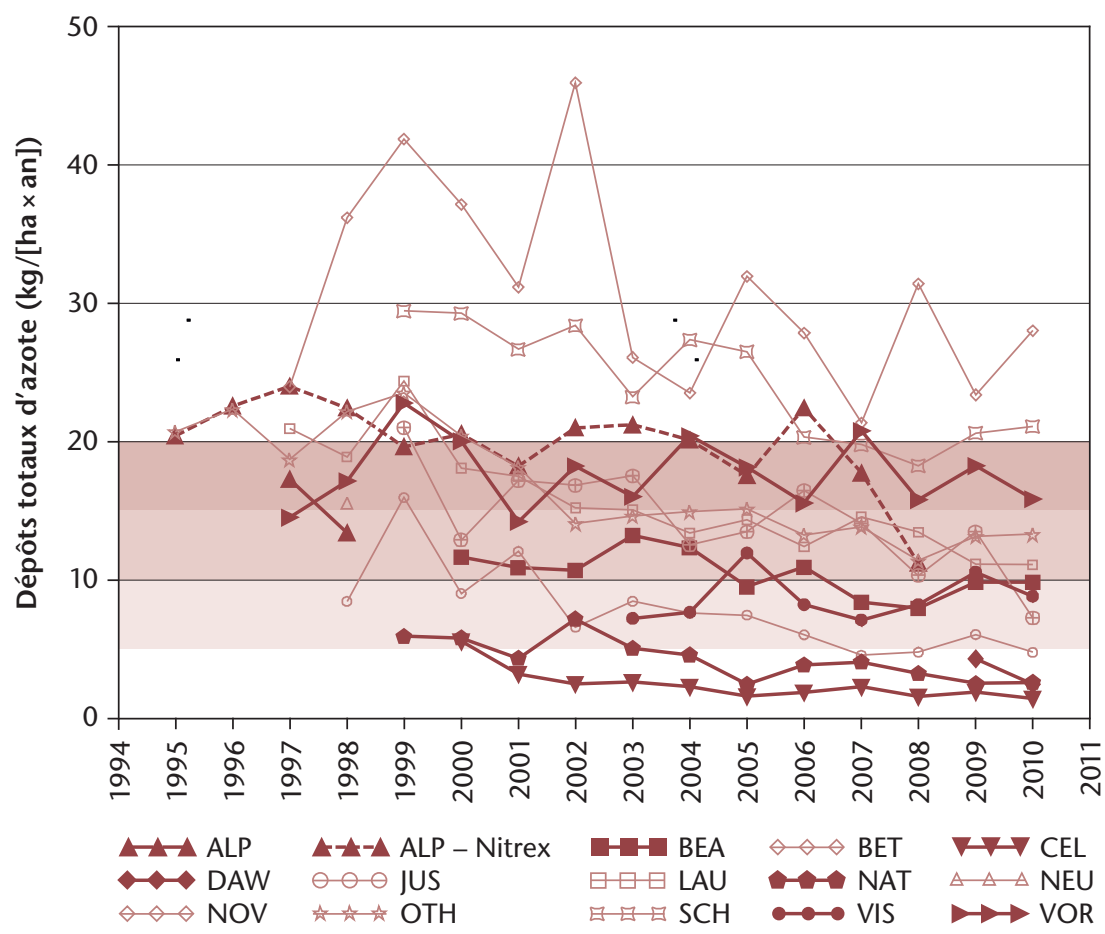

Fig. 2 Evolution des dépôts totaux d'azote annuels sur les sites LWF et à Alptal, estimés par un modèle de bilan appliqué aux pluviolessivats. Chaque site LWF est désigné par un code qui correspond aux trois premières lettres du nom du site indiqué dans la figure 1. Symboles vides: peuplements de feuillus, symboles pleins: peuplements de conifères. Les rectangles ambrés indiquent la gamme des charges critiques empiriques (5 à $15 \mathrm{~kg} \mathrm{~N}$ / [ha $\times$ an] pour les conifères, 10 à $20 \mathrm{~kg} \mathrm{~N} /[\mathrm{ha} \times$ an] pour les feuillus).

la quantité d'eau de percolation calculée par le modèle COUP (Jansson \& Karlberg 2004, voir aussi Graf Pannatier et al 2011).

A Alptal, la parcelle témoin étant un petit bassin versant, le lessivage est mesuré directement à l'exutoire de ce bassin, par analyse toutes les deux semaines d'échantillons d'eau d'écoulement prélevés automatiquement et proportionnellement au débit (Schleppi et al 2006b).

\section{Concentrations foliaires}

Le feuillage est échantillonné tous les quatre ans sur les sites IAP depuis 1984, tous les deux ans sur 17 des 18 sites LWF depuis 1995-1997, tous les ans à Alptal depuis 1994. Des branches sont prélevées dans le tiers supérieur de la couronne d'arbres appartenant à la strate arborée dominante ou co-dominante, au nombre de 8 sur les sites IAP, 5 à 6 sur les sites LWF et à Alptal. Dans le cas des conifères, les pousses sont séparées en aiguilles de l'année en cours (c) et aiguilles de l'année précédente $(c+1)$. Feuilles et aiguilles séparées par classes d'âge sont séchées à $65^{\circ} \mathrm{C}$, broyées, puis analysées en laboratoire (Schleppi et al 1999; Thimonier et al 2010). Sur les sites IAP, seules les aiguilles âgées d'un an et demi $(c+1)$ sont analysées. Les concentrations mesurées peuvent être comparées à des valeurs de référence, déterminées sur la base de relations entre croissance des arbres et concentrations foliaires établies dans le cadre d'essais de fertilisation. Ces concentrations de référence in- diquent si un élément nutritif, en concentration absolue ou relative par rapport à un autre élément, est disponible en quantité suffisante.

\section{Végétation du sous-bois}

Sur 43 sites IAP, les plantes de la strate herbacée présentes sur des sous-placettes délimitées par des arbres numérotés ont été inventoriées dans les périodes 1984/85 et 2003/04. Le recouvrement de chaque espèce a été estimé selon la méthode de Braun-Blanquet (Braun-Blanquet 1964).

Sur 16 sites LWF, la flore du sous-bois est inventoriée depuis la période 1994-1998 sur 16 carrés permanents de $1 \mathrm{~m}^{2}$ ainsi que sur des placettes circulaires de $500 \mathrm{~m}^{2}$ de surface (Thimonier et al 2011). Sur les carrés permanents, le recouvrement de chaque espèce dans les strates herbacée et muscinale est estimé visuellement en pourcentage, tandis que sur les placettes circulaires, l'abondance-dominance des espèces est quantifiée selon la méthode de Braun-Blanquet. La dernière campagne de relevés a eu lieu au cours de l'été 2011.

Le caractère nitrophile d'une plante peut être exprimé sous forme d'indice, en utilisant le coefficient $\mathrm{N}$ d'Ellenberg (Ellenberg et al 1992), ou le coefficient $\mathrm{N}$ de Landolt (Landolt 1977), identiques dans leur principe. Les exigences ou préférences de chaque espèce à l'égard de l'azote sont codées sur une échelle de 1 à 9 (Ellenberg) ou de 1 à 5 (Landolt), les valeurs basses étant affectées aux espèces préférant un milieu pauvre en azote, les valeurs hautes aux espèces exigeant des milieux riches.

Nous avons calculé pour chaque site un indice $\mathrm{N}$ moyen correspondant à la moyenne non pondérée du coefficient $\mathrm{N}$ affecté à chacune des espèces présentes dans un relevé à une date donnée. Nous avons ensuite identifié d'éventuels changements en comparant les valeurs obtenues pour les relevés de la première campagne de relevés (de 1983 à 1984 pour le réseau IAP, de 1994 à 1998 pour le réseau LWF) à celles du dernier inventaire (IAP: de 2003 à 2004, LWF: en 2011).

\section{Méthodes statistiques}

Afin de détecter les tendances dans la variation temporelle des dépôts atmosphériques annuels, nous avons appliqué à chaque site LWF et à Alptal le test de Mann-Kendall, à l'aide du programme MULTMK/PARTMK1. Nous avons également appliqué des tests partiels afin de déterminer si les variations temporelles détectées pouvaient être attribuées à des fluctuations de la pluviométrie annuelle, un paramètre assez bien corrélé aux dépôts (Thimonier et al 2005). Nous avons appliqué le même test pour évaluer les tendances pour le lessivage de nitrate.

\footnotetext{
1 LIBISELLER C (2004) MULTMK/PARTMK, a program for the computation of Multivariate and Partial Mann-Kendall Test. Available online at http://wwwekonsluse/PMK (25.8.2009).
} 
Pour les concentrations foliaires, nous avons d'abord recherché la présence de tendances dans les concentrations foliaires en utilisant de simples régressions linéaires sur le temps pour chaque site LWF pris individuellement et à Alptal. Les tendances ont également été testées à l'aide de modèles linéaires mixtes, en regroupant les sites selon l'essence principale. Pour les sites LWF, la procédure MIXED sous SAS a été appliquée pour le hêtre, l'épicéa + sapin, et le pin (Jonard et al 2009). Pour les sites IAP, la fonction «lme» sous SPLUS a été utilisée pour le hêtre et pour l'épicéa. Les corrélations entre paramètres ont été testées à l'aide de régressions mixtes multivariées.

L'évolution de la végétation du sous-bois sur les sites LWF et IAP a été évaluée à l'aide de tests pour échantillons appariés.

\section{Résultats}

\section{Dépôts atmosphériques}

Sur les sites LWF et à Alptal, les dépôts totaux d'azote estimés par un modèle de bilan appliqué aux pluviolessivats varient entre $3 \mathrm{~kg} \mathrm{~N} /($ ha $\times$ an) (moyenne à Celerina, dans les Alpes) et $31 \mathrm{~kg} \mathrm{~N} /$ (ha $\times$ an) (moyenne à Novaggio, au Tessin). Les dépôts d'azote sont également élevés à Schänis, site situé sur le contrefort nord des Alpes (24 kg N/[ha×an] en moyenne). A Novaggio et à Schänis (peuplements de feuillus), les dépôts excèdent la charge critique empirique maximale de $20 \mathrm{~kg} \mathrm{~N} /($ ha $\times$ an) pour l'azote. Sur le Plateau (à l'exception de Jussy) et dans le Jura, les dépôts d'azote se situent entre les charges critiques minimale et maximale (10 et $20 \mathrm{~kg} \mathrm{~N} /[$ ha $\times$ an], respectivement, pour les feuillus). Dans les peuplements de conifères situés dans les Alpes, ils sont inférieurs à la charge critique minimale de $5 \mathrm{~kg} \mathrm{~N} /($ ha $\times$ an) (figure 2). A Alptal, les dépôts azotés s'élèvent à $20 \mathrm{~kg}$ $\mathrm{N} /($ ha $\times$ an) en moyenne et excèdent ainsi les charges critiques pour les conifères.

La variabilité interannuelle des dépôts azotés est grande, et elle est en partie liée à la pluviométrie des années de mesure. Une tendance à la baisse se dessine pour une partie des sites. Elle est significative sur les sites du Plateau (sauf Vordemwald), sur les sites d'altitude (Parc National, Celerina), et sur le contrefort nord des Alpes (Schänis) (figure 2, tableau 1). A Bettlachstock, dans le Jura, les dépôts di-

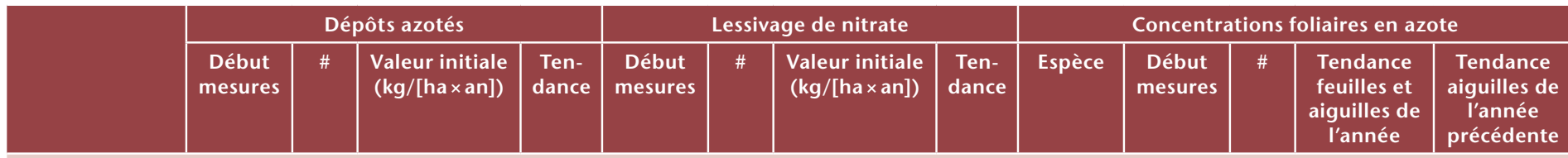

Jura

\begin{tabular}{|c|c|c|c|c|c|c|c|c|c|c|c|c|c|}
\hline \multirow[t]{2}{*}{ Bettlachstock } & 1999 & 12 & 21.0 & $=$ & 2001 & 9 & 3.1 & $=$ & Hêtre & 1997 & 7 & $=$ & \\
\hline & & & & & & & & & Sapin & 1995 & 8 & $=$ & $(\downarrow)$ \\
\hline Neunkirch & & & & & & & & & Hêtre & 1997 & 7 & $=$ & \\
\hline \multicolumn{14}{|l|}{ Plateau Suisse } \\
\hline Jussy & 1998 & 13 & 8.5 & $\downarrow$ & & & & & Chêne & 1997 & 7 & $=$ & \\
\hline Lausanne & 1997 & 14 & 20.4 & $\downarrow \downarrow$ & 2000 & 10 & 23.1 & $=$ & Hêtre & 1997 & 7 & $=$ & \\
\hline Othmarsingen & 1995 & 16 & 20.7 & $\downarrow \downarrow$ & & & & & Hêtre & 1997 & 7 & $\downarrow \downarrow$ & \\
\hline Vordemwald & 1997 & 14 & 14.5 & $=$ & 2000 & 10 & 0.4 & $\downarrow$ & Sapin & 1995 & 8 & $=$ & $=$ \\
\hline \multicolumn{14}{|l|}{ Préalpes } \\
\hline Schänis & 1999 & 12 & 29.4 & $(\downarrow)$ & 2001 & 9 & 15.2 & $=$ & Hêtre & 1999 & 6 & $\downarrow$ & \\
\hline Beatenberg & 2000 & 11 & 11.7 & $(\downarrow)$ & 2001 & 9 & 0.5 & $=$ & Epicéa & 2001 & 7 & $=$ & $=$ \\
\hline Alptal NITREX & 1995 & 14 & 20.4 & $(\downarrow)$ & 1997 & 11 & 2.5 & $(\downarrow)$ & Epicéa & 1995 & 14 & $=$ & (ע) \\
\hline Alptal LWF & & & & & & & & & Epicéa & 1995 & 8 & $=$ & $=$ \\
\hline
\end{tabular}

Alpes

\begin{tabular}{|c|c|c|c|c|c|c|c|c|c|c|c|c|c|}
\hline Celerina & 2000 & 11 & 5.6 & $(\downarrow)$ & 2001 & 9 & 0.1 & $(\downarrow)$ & Pin & 1997 & 7 & $=$ & $=$ \\
\hline Parc National & 1999 & 12 & 5.9 & $(\downarrow)$ & & & & & Pin & 1997 & 7 & $=$ & $(\downarrow)$ \\
\hline Lens & & & & & & & & & Pin & 1997 & 7 & $=$ & $(\downarrow)$ \\
\hline Visp (Viège) & 2003 & 8 & 7.9 & $=$ & & & & & Pin & 1997 & 7 & $=$ & $(\downarrow)$ \\
\hline \multicolumn{14}{|l|}{ Tessin } \\
\hline Chironico & & & & & & & & & Epicéa & 1997 & 7 & $=$ & $=$ \\
\hline Isone & & & & & & & & & Hêtre & 1997 & 7 & $(\downarrow)$ & \\
\hline Novaggio & 1997 & 14 & 24.0 & $=$ & 2001 & 9 & 2.7 & $=$ & Chêne & 1997 & 7 & $=$ & \\
\hline
\end{tabular}

Tab. 1 Tendances temporelles observées sur les sites LWF et à Alptal pour les dépôts totaux d'azote estimés par un modèle de bilan appliqué aux pluviolessivats (test de Mann-Kendall éliminant l'effet de la variation temporelle des précipitations), le lessivage de nitrate, et les concentrations foliaires en azote. $=$ pas de tendance, $(\mathbf{\nabla})$ tendance à la diminution $(p<0.10),(\boldsymbol{\downarrow})$ diminution significative $(p<0.05)$, $\boldsymbol{\downarrow}$ diminution significative $(p<0.01), \boldsymbol{\downarrow}$ diminution très significative $(p<0.001)$, \# nombre d'années de mesure. 


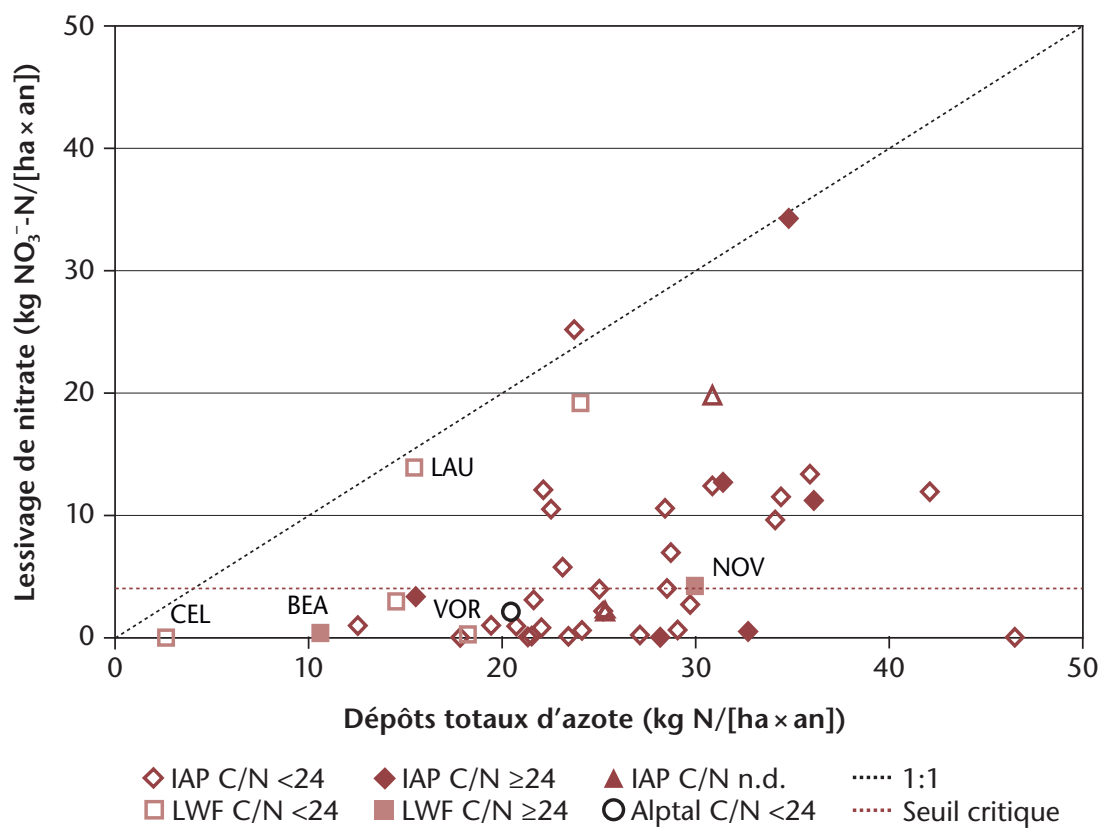

Fig. 3 Relation entre le lessivage de nitrate et les dépôts totaux d'azote estimés par un modèle de bilan appliqué aux pluviolessivats (LWF, Alptal) ou modélisés (IAP). Les sites sont classés en fonction du réseau et du rapport $C / N$ de l'horizon organique du sol (n.d.: non déterminé). Sites LWF: lessivage et dépôts moyens pour la période >1999-2009 $(n=7)$; Alptal: lessivage et dépôts moyens pour 1997-2007; sites IAP: lessivage moyen pour la période 2003-2007 $(n=40)$. Le seuil critique est de $2-4 \mathrm{~kg} \mathrm{~N} /($ ha $\times$ an). Seule la limite supérieure de ce seuil est représentée.

minuent significativement, mais cette tendance n'est plus apparente si l'effet de la variation temporelle des précipitations est pris en compte.

Nous constatons une diminution significative des flux d'ammonium dans les pluviolessivats sur tous les sites LWF du Plateau à l'exception de Vordemwald, sur les deux sites d'altitude - Celerina et le Parc National - et à Novaggio (résultats non montrés). Les flux de nitrate dans les pluviolessivats diminuent sur tous les sites LWF à l'exception de Vordemwald et de Novaggio.

\section{Lessivage de nitrate}

Le lessivage de nitrate mesuré sur les sites LWF varie largement d'un site à l'autre. Les flux de nitrate sont pratiquement nuls à Celerina, Vordemwald et Beatenberg. En moyenne, ils atteignent $13 \mathrm{~kg}$ $\mathrm{N} /($ ha $\times$ an) à Lausanne et $19 \mathrm{~kg} \mathrm{~N} /($ ha $\times$ an) à Schänis (figure 3). Seuls ces deux sites connaissent des flux de nitrate dépassant régulièrement le seuil des $4 \mathrm{~kg}$ $\mathrm{N} /($ ha $\times$ an). En revanche, au sein du réseau IAP, le lessivage de nitrate excède $4 \mathrm{~kg} \mathrm{~N} /($ ha $\times$ an) sur 19 des 40 sites échantillonnés (figure 3 ).

Les mesures effectuées sur les sites LWF révèlent de larges variations interannuelles des flux de nitrate, notamment à Lausanne et Schänis. Ces variations sont déterminées en partie par les variations de flux d'eau. A Schänis, par exemple, le lessivage de nitrate a atteint un maximum de $44 \mathrm{~kg} / \mathrm{ha}$ en 2002 , année pour laquelle les précipitations étaient 30\% plus élevées que la moyenne des précipitations mesurées pour les autres années de mesures.
Nous n'avons détecté de tendances dans l'évolution temporelle du lessivage de nitrate sur aucun des sites LWF, sauf à Vordemwald, où les flux de nitrate, $<1 \mathrm{~kg} \mathrm{~N} /$ ha en 2000 , ont tendance à diminuer. A Alptal, le lessivage de nitrate diminue significativement de 1997 à 2007.

L'intensité du lessivage de nitrate sur un site dépend de l'importance des dépôts atmosphériques (figure 3). Sur certains sites, le flux de nitrate est proche de la valeur des dépôts. Certains sites reçoivent cependant d'importants dépôts azotés sans pour autant que le lessivage de nitrate ne soit élevé. Parmi les sites LWF, c'est le cas de Novaggio. La figure 3 distingue les sites en fonction de la valeur du rapport $\mathrm{C} / \mathrm{N}$ de l'horizon organique du sol, un indicateur de la disponibilité en azote pour les plantes et les micro-organismes. Les classes de valeurs $\mathrm{C} / \mathrm{N}$ $(\mathrm{C} / \mathrm{N}<24$ et $\mathrm{C} / \mathrm{N} \geq 24)$ ont été choisies sur la base des travaux de Dise et al (2009), qui observent des pertes de nitrate par lessivage plus élevées quand le rapport $\mathrm{C} / \mathrm{N}$ est plus faible, indiquant que l'azote est facilement disponible. A Novaggio (NOV, figure 3), le rapport $\mathrm{C} / \mathrm{N}$ est relativement élevé, et malgré des dépôts importants, le lessivage de nitrate est limité. Sur les sites IAP, les premières analyses statistiques ne détectent pas d'influence du rapport $\mathrm{C} / \mathrm{N}$.

\section{Analyses foliaires}

Les concentrations foliaires en azote mesurées chez le hêtre indiquent une qualité de nutrition azotée satisfaisante pour l'ensemble des peuplements des sites IAP et LWF, voire un surplus d'azote par rapport aux besoins nutritionnels des arbres: certains sites présentent des concentrations plus élevées que la limite supérieure de la gamme de valeurs satisfaisantes. Les concentrations foliaires azotées ont diminué significativement au cours du temps depuis le début des mesures (1997 pour les sites LWF, 1984 pour les sites IAP; figure $4 \mathrm{a}$ ).

Chez les conifères à petites aiguilles (épicéa et sapin), les concentrations en azote se situent dans la gamme de valeurs satisfaisantes pour la nutrition en cet élément ou en dessous de la limite inférieure de cette gamme (figure $4 \mathrm{~b}$ ). Nous n'observons pas de tendance temporelle nette pour les concentrations mesurées sur les aiguilles de l'année courante (sites LWF et Alptal), mais les concentrations déterminées sur les aiguilles âgées d'un an et demi (c+1) diminuent significativement sur les sites IAP, et tendent à diminuer sur certains sites LWF considérés individuellement. Sur le site NITREX d'Alptal (épicéa), cette tendance à la diminution semble également être présente pour les aiguilles $c+1$, de façon peu marquée $(p=0.066)$. Ces tendances générales à la baisse sont confirmées par les modèles linéaires mixtes appliqués sur les sites LWF et IAP par groupe d'espèces (tableau 2).

Les concentrations foliaires en phosphore chez le hêtre indiquent que cet élément est en quantité 

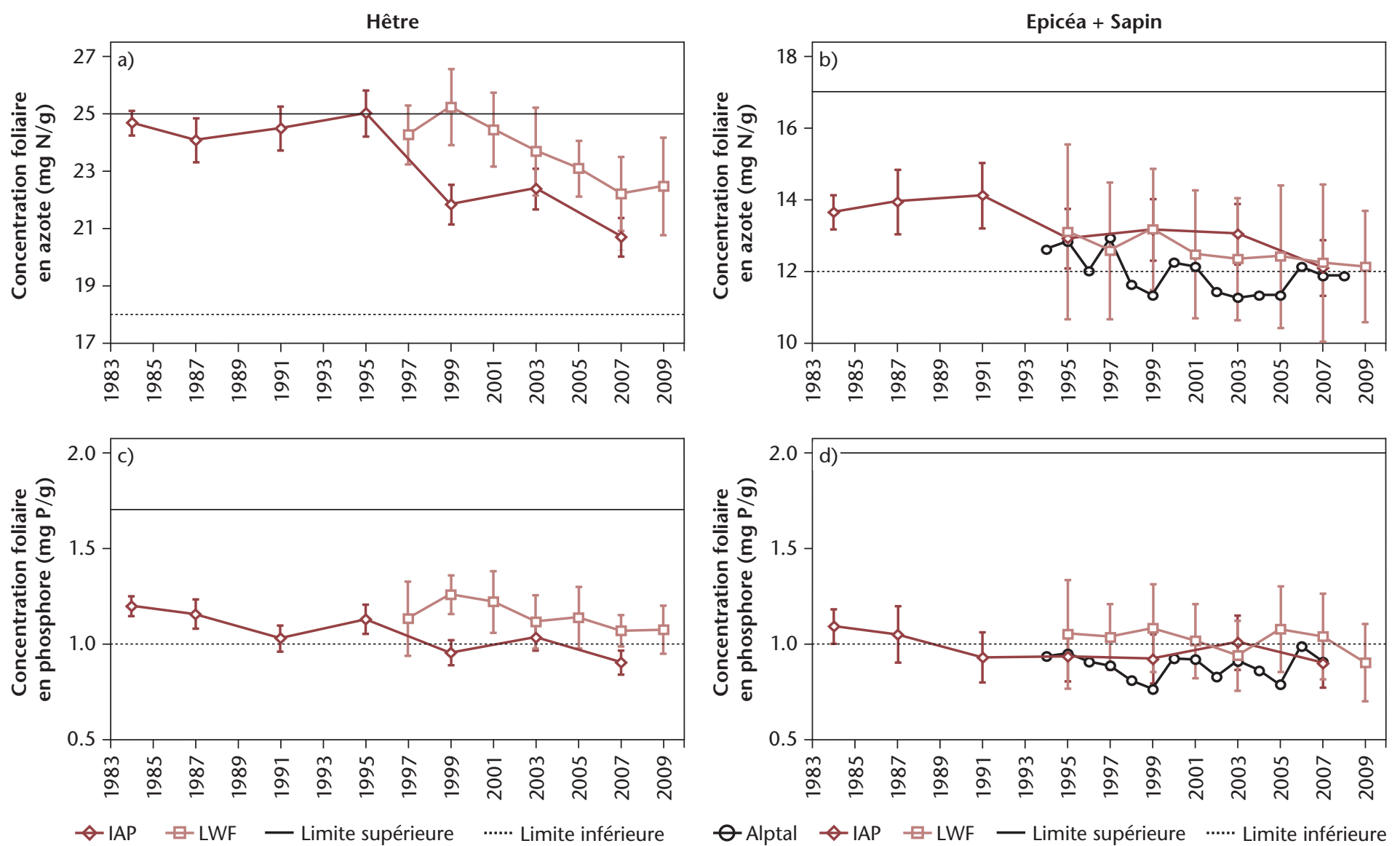

Fig. 4 Variation temporelle des concentrations foliaires chez le hêtre (à gauche) et dans les aiguilles âgées d'un an et demi (c + 1) chez l'épicéa + sapin (à droite) sur les sites d'Alptal, IAP et LWF. La moyenne des sites au sein de chacun des deux réseaux IAP et LWF est représentée; les barres indiquent I'intervalle de confiance à 95\%. Les deux lignes horizontales (en pointillé pour la limite inférieure, en trait plein pour la limite supérieure) délimitent la gamme de concentrations pour laquelle la nutrition en l'élément considéré est satisfaisante (Stefan et al 1997). a) et b): concentrations en azote N. c) et d): concentrations en phosphore $P$.

suffisante, bien que dans le domaine inférieur de la gamme de concentrations optimales, sur les sites LWF, à l'exception de Bettlachstock (figure 4c). Sur les sites IAP, la concentration moyenne des peuplements étudiés est, en 2007, plus basse que la limite inférieure définissant la gamme de valeurs optimales. Sur ces sites, les concentrations en phosphore ont diminué significativement depuis 1984. En 1984, seuls $12 \%$ des peuplements de hêtre IAP présentaient une nutrition insuffisante en phosphore. Ce chiffre passe à 71\% en 2007 (Flückiger et al 2011). Sur le ré- seau LWF, des régressions linéaires sur les années de mesure appliquées site par site ne révèlent pas de tendance nette. Cependant, si l'on considère l'ensemble des sites en appliquant des modèles linéaires mixtes, on observe une légère tendance à la diminution (tableau 2).

En ce qui concerne l'épicéa, la proportion de peuplements pour lesquels les concentrations foliaires en phosphore indiquent une carence en cet élément passe de 6\% en 1984 à 66\% en 2007 dans le réseau IAP (Flückiger et al 2011). Au sein du réseau

\begin{tabular}{|c|c|c|c|c|c|c|c|c|c|c|}
\hline & & \multicolumn{3}{|c|}{ Concentration foliaire en azote $(\mathrm{N})$} & \multicolumn{3}{|c|}{ Concentration foliaire en phosphore $(P)$} & \multicolumn{3}{|c|}{ Rapport foliaire N/P } \\
\hline & & LWF & Alptal & IAP & LWF & Alptal & IAP & LWF & Alptal & IAP \\
\hline Hêtre & & $\downarrow$ & & $\downarrow \downarrow$ & (ע) & & $\downarrow \downarrow$ & $=$ & & 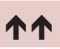 \\
\hline \multirow[t]{2}{*}{ Epicéa (+ sapin) } & aiguilles c & $=$ & $=$ & & $(\Downarrow)$ & $=$ & & $(\pi)$ & $=$ & \\
\hline & aiguilles $c+1$ & $\downarrow$ & (ע) & $\downarrow \downarrow$ & (ע) & $=$ & $\downarrow$ & $=$ & $=$ & $(\boldsymbol{\uparrow})$ \\
\hline Pin & aiguilles $c+1$ & $(\Downarrow)$ & & & $\downarrow$ & & & $=$ & & \\
\hline
\end{tabular}

Tab. 2 Résultat des modèles linéaires mixtes appliqués sur les sites LWF et IAP et de régressions linéaires appliquées au site d'Alptal. = pas de tendance; ( $\mathbf{(})$ tendance à la diminution $(p<0.10)$, ( $\boldsymbol{(})$ tendance à l'augmentation $(p<0.10)$, ( $\boldsymbol{\Downarrow})$ diminution significative $(p<0.05)$, ( $\boldsymbol{\uparrow})$ augmentation significative

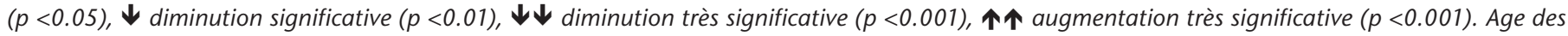
aiguilles: «aiguilles $c »$ : aiguilles de l'année en cours; «aiguilles $c+1$ »: aiguilles de l'année précédente. 

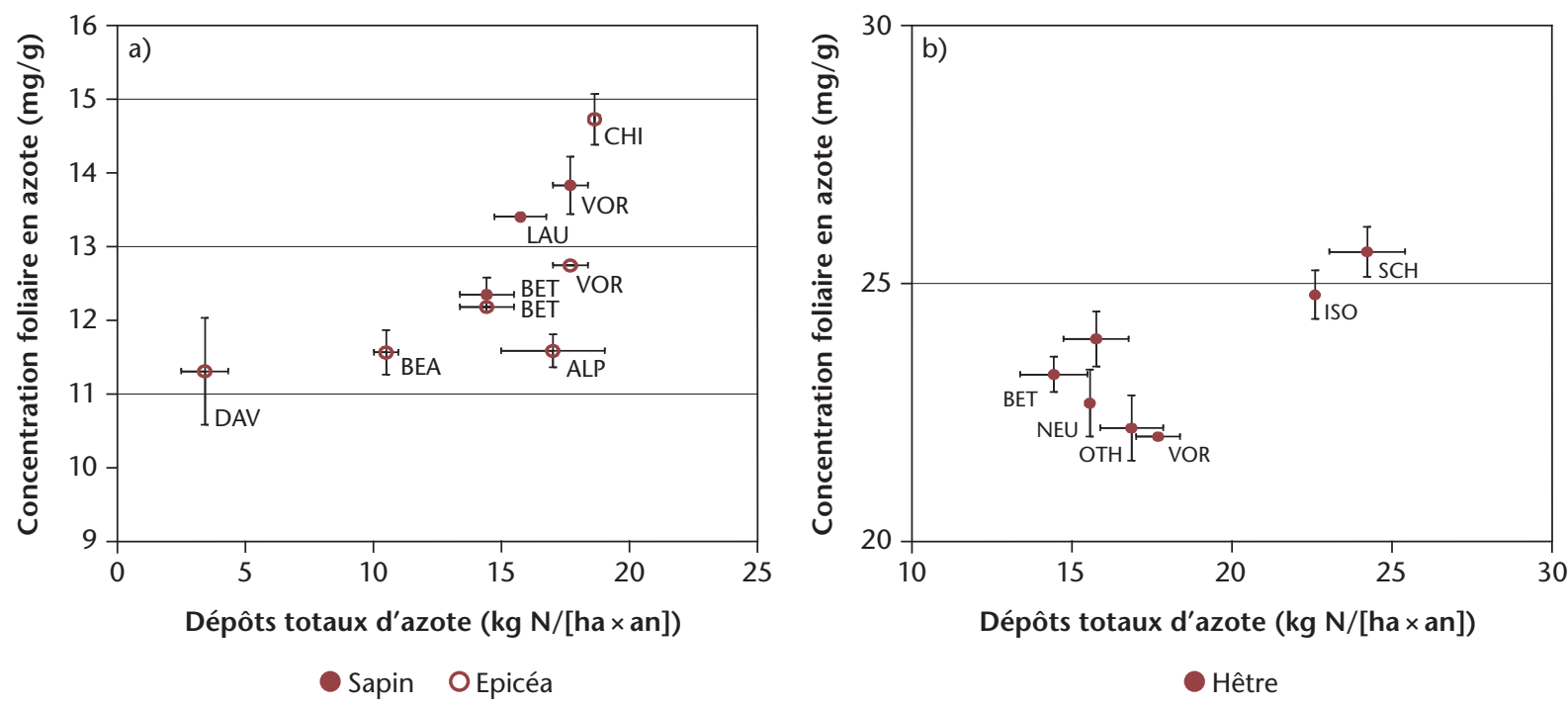

Fig. 5 Relation entre les concentrations foliaires en azote et les dépôts totaux d'azote annuels sur les sites LWF. a) épicéa et sapin (aiguilles de l'année en cours); b) hêtre. Les points représentent la moyenne sur toutes les années de mesure, les barres représentent l'erreur type. Les dépôts totaux d'azote correspondent aux dépôts estimés par un modèle de bilan appliqué aux pluviolessivats, sauf pour Chironico (CHI) et Isone (ISO), sites pour lesquels les dépôts sont modélisés.

LWF, les concentrations en phosphore mesurées sur les aiguilles âgées d'un an et demi se situent, comme chez le hêtre, autour de la limite inférieure de la gamme de concentrations optimales (figure $4 \mathrm{~d}$ ). Les concentrations en phosphore mesurées sur les aiguilles de l'année tendent cependant à être plus élevées (résultat non montré). L'application de modèles linéaires mixtes à l'ensemble des sites LWF avec épicéa ou sapin révèle une tendance à la diminution des concentrations en phosphore depuis le début des mesures en 1995/1997 (tableau 2). Cette diminution est également observée, de manière significative, sur les sites IAP. En revanche, à Alptal, nous ne détectons pas de tendance à la baisse.

Le rapport de concentrations foliaires N/P, un autre indicateur de la qualité de la nutrition des arbres, reste relativement stable au cours de la période de mesure chez l'épicéa (réseau LWF et Alptal) et chez le hêtre du réseau LWF (tableau 2). En revanche, le rapport N/P a augmenté chez l'épicéa et surtout chez le hêtre au sein du réseau IAP (tableau 2). Chez le hêtre, le rapport N/P excède 17 sur une majorité de sites, et est ainsi au-dessus des valeurs recommandées sur la base d'une compilation d'études (Flückiger \& Braun 2003). Chez l'épicéa, il se situe, selon les sites, dans la gamme de valeurs optimales ou au-dessus de la limite supérieure fixée à 12. En moyenne pour le réseau IAP, le rapport N/P chez l'épicéa excède nettement, comme pour le hêtre, la valeur limite supérieure.

Les concentrations en azote dans les aiguilles d'épicéa (et de sapin) sont positivement corrélées aux dépôts atmosphériques d'azote sur les sites LWF (figure $\left.5 \mathrm{a} ; \mathrm{R}^{2}=0.54, \mathrm{p}<0.05\right)$ et $\mathrm{IAP}\left(\mathrm{R}^{2}=0.18, \mathrm{p}<0.001\right)$. Il semble y avoir une relation similaire pour les peuplements de hêtre du réseau LWF (figure $5 \mathrm{~b} ; \mathrm{R}^{2}=0.57$, $\mathrm{p}<0.05)$, mais pas pour les hêtraies du réseau IAP.
Cependant, lorsque les concentrations foliaires sont corrigées afin de soustraire l'effet de l'altitude, la relation avec les dépôts n'est plus significative.

\section{Changements de la végétation du sous-bois}

Sur les sites IAP, la comparaison de relevés de végétation effectués en 1984 et en 2003/2004 révèle une tendance significative à l'augmentation de la valeur indicatrice d'Ellenberg pour l'azote, exprimant une augmentation du caractère nitrophile de la végétation (figure 6a). Sur les sites LWF, sur une période plus récente et plus courte (13-17 ans), nous n'observons pas de changement significatif de l'indice d'azote (figure 6b).

\section{Discussion et conclusion}

En l'espace d'une quinzaine d'années, les apports d'azote d'origine atmosphérique ont diminué significativement sur huit des douze sites analysés en continu (LWF + Alptal). Ces résultats concordent avec la réduction des émissions d'azote enregistrée en Suisse au cours des deux dernières décennies (Heldstab et al 2010), mais ils montrent aussi que cette réduction n'est pas aussi générale que celle du soufre (Graf Pannatier et al 2012). De même, le Réseau national d'observation des polluants atmosphériques permet de mettre en évidence une diminution significative du taux d'oxydes d'azote $\left(\mathrm{NO}_{\mathrm{X}}\right)$ dans l'air depuis 1990 (OFEV 2011), mais n'observe pas de diminution des apports d'azote $\left(\mathrm{NH}_{4}{ }^{+}+\mathrm{NO}_{3}{ }^{-}\right)$ par la pluie au cours du temps sur ses stations de mesure. Nous observons sur nos sites une diminution des apports de $\mathrm{NH}_{4}{ }^{+}$dans les pluviolessivats pratiquement identique à celle des apports de $\mathrm{NO}_{3}{ }^{-}$. Nos mesures ne reflètent donc pas la diminution plus 


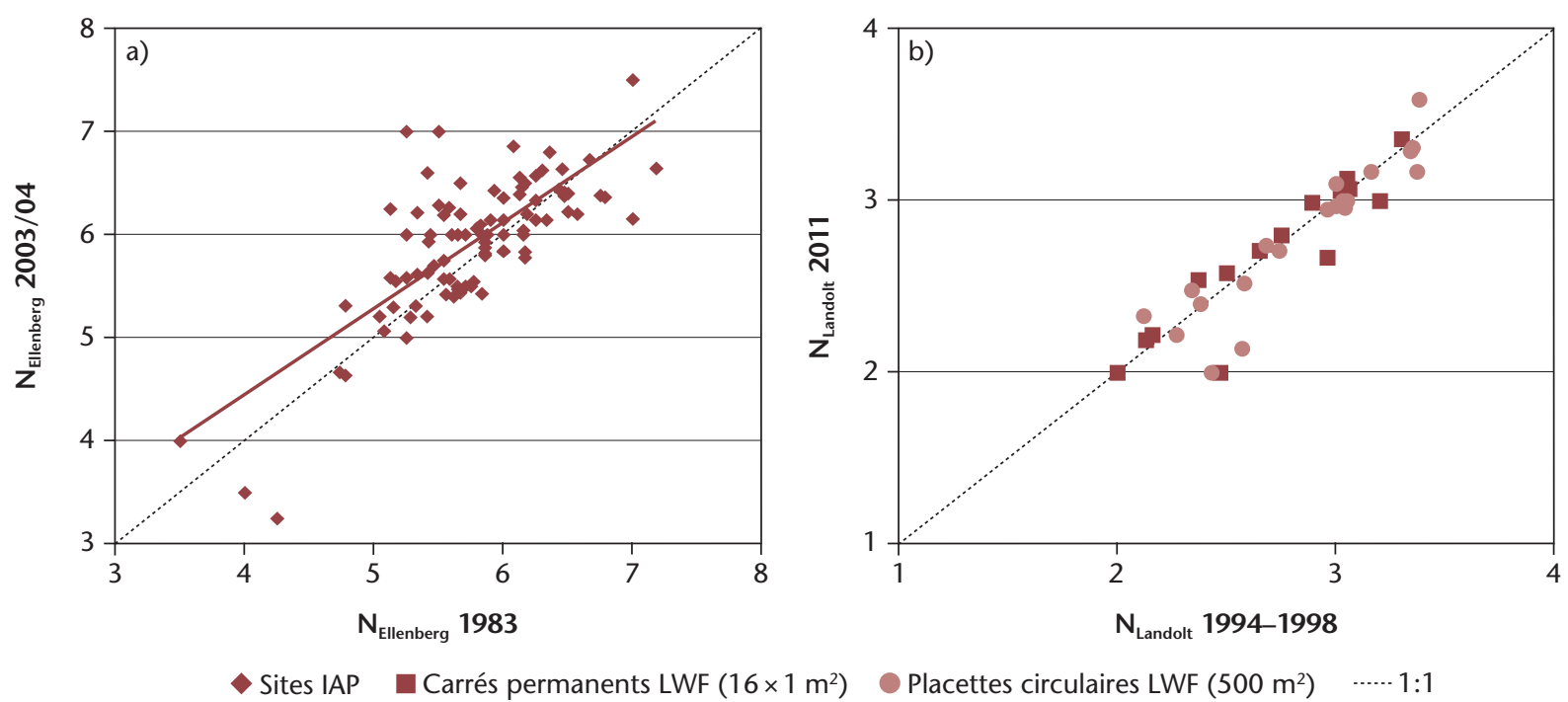

Fig. 6 a) Valeurs indicatrices d'Ellenberg pour l'azote calculées par site IAP sur les relevés effectués en 2003/2004, en relation avec les valeurs indicatrices des relevés effectués en 1983/1984; b) Valeurs indicatrices de Landolt pour l'azote calculées par site LWF sur les relevés effectués en 2011 en relation avec les valeurs indicatrices des premiers relevés (moyenne 1994-1998).

nette des émissions de $\mathrm{NO}_{\mathrm{x}}$ comparées à celles de $\mathrm{NH}_{3}$. Il est possible que des transformations microbiennes des composés azotés à l'intérieur des collecteurs au cours de la période d'échantillonnage modifient les proportions relatives de $\mathrm{NO}_{3}{ }^{-}$et $\mathrm{NH}_{4}{ }^{+}$. Comme les $\mathrm{NO}_{\mathrm{X}}$ sont transportés sur de plus grandes distances dans l'atmosphère que le $\mathrm{NH}_{3}$, il se peut également que les dépôts soient encore largement influencés par les émissions dans le reste de l'Europe, qui toutefois diminuent également.

Malgré le déclin observé sur certains sites, les dépôts actuels restent proches des charges critiques empiriques ou les dépassent sur une majorité de sites. C'est d'autant plus vrai si l'on considère la sous-estimation des dépôts totaux quantifiés à l'aide du modèle appliqué aux pluviolessivats. Le lessivage de nitrate, un des effets négatifs qui peuvent se produire lorsque les dépôts excèdent les charges critiques, se situe dans la gamme de valeurs rapportée par de Vries et al (2007) sur d'autres sites européens (119 sites entre 0 et $20 \mathrm{~kg} \mathrm{~N} /[\mathrm{ha} \times \mathrm{an}]$ et deux sites entre 20 et $50 \mathrm{~kg} \mathrm{~N} /[$ ha×an]). Le lessivage n'excède régulièrement la valeur seuil de $4 \mathrm{~kg}$ que sur deux des sept sites LWF étudiés: à Lausanne, où les dépôts se situent dans le domaine des charges critiques, et à Schänis, où les dépôts excèdent ces charges. Le lessivage est supérieur à $4 \mathrm{~kg} \mathrm{~N} /($ ha $\times$ an) sur une plus grande proportion de sites IAP (19 sur 40). Comme d'autres études l'ont montré, nous observons une relation entre dépôts azotés et lessivage. Cette relation est en partie modulée par les propriétés du sol telles que le rapport $\mathrm{C} / \mathrm{N}$ de l'horizon organique, indicateur de la disponibilité en azote. En effet, les forêts dont le sol possède un horizon organique avec un rapport $\mathrm{C} / \mathrm{N}<24$ relâchent davantage de nitrate que celles ayant un rapport $>24$ (Dise et al 2009). Nous avons pu confirmer qualitativement cette hypothèse sur les sites LWF (Thimonier et al 2010). Le nombre de sites LWF est cependant insuffisant pour tester statistiquement ces relations. Sur le jeu de données plus étoffé que constituent les 40 sites IAP, les 7 sites LWF et le site d'Alptal, le rapport C/N apparaît comme un indicateur médiocre, comme cela avait déjà été constaté sur 57 sites du réseau d'observation allemand (Borken \& Matzner 2004). Le fait que les sites avec un rapport $\mathrm{C} / \mathrm{N}>24$ soient très peu représentés dans notre jeu de données contribue à rendre difficile la mise en évidence de telles relations.

A Alptal, les dépôts azotés diminuent significativement et le lessivage de nitrate également. En revanche, à Lausanne et à Schänis, les dépôts diminuent significativement, mais nous n'observons pas de tendance à la baisse pour le lessivage, compte tenu de la forte variation interannuelle des flux de nitrate quittant l'espace racinaire. De plus, le nitrate quittant l'espace racinaire est le résultat net de plusieurs processus (absorption du nitrate par les racines, nitrification, immobilisation) et ne reflète pas directement les apports atmosphériques d'azote. Le sol est une source différée de nitrate, lorsque l'azote déposé, d'abord retenu dans le sol, est ensuite minéralisé à moyen ou long terme. Ce processus pourrait également expliquer pourquoi le caractère nitrophile de la végétation du sous-bois a augmenté entre 1984 et 2003/2004 sur les sites IAP, alors que le réseau IAP a été mis en place dans une période où les émissions de polluants azotés atteignaient un pic et qu'un déclin relatif s'amorçait. Les résultats du réseau IAP concordent avec ceux d'autres études suisses (Kuhn et al 1987) et européennes (Thimonier et al 1992; Diekmann et al 1999) qui montrent également une eutrophisation du milieu. L'absence de changements floristiques significatifs pour les sites LWF peut s'expliquer par la période à la fois plus courte et plus ré- 
cente sur laquelle les relevés anciens et récents ont été comparés, par le plus petit nombre de sites (16), ou par l'interférence d'autres facteurs prépondérants pour la dynamique de la végétation herbacée, comme les changements de lumière (Thimonier et al 2011).

Alors que la végétation du sous-bois indique une disponibilité plus élevée (IAP) ou inchangée (LWF) de l'azote dans le milieu, les analyses foliaires des réseaux IAP et LWF révèlent une tendance à la diminution des concentrations en azote du feuillage des arbres au cours des 15-25 dernières années. Notons que pour les conifères, cette tendance est essentiellement visible sur les aiguilles de l'année précédente. Les aiguilles âgées d'un an et demi reflètent mieux le statut nutritif des arbres que les aiguilles de l'année courante, sans doute à cause des processus de re-translocation d'éléments (Braekke \& Salih 2002). D’autres études portant sur une période antérieure ont montré une tendance contraire à la hausse des concentrations foliaires d'azote (Duquesnay et al 2000 dans des hêtraies entre 1969-71 et 1996-97; Mellert et al 2004 dans des peuplements de pin sylvestre d'Europe centrale entre 1964 et 1999). La tendance à la baisse de la concentration foliaire en azote constatée par Mellert et al (2004) dans des peuplements d'épicéa a été attribuée par les auteurs à un effet de dilution, indiqué par l'augmentation du poids des aiguilles. Nous n'observons pas une telle tendance pour le poids des aiguilles ou des feuilles sur nos sites. Il n'est donc pas exclu que la diminution des concentrations foliaires en azote soit liée à la réduction concomitante des dépôts azotés, qui semblent jouer un rôle dans la composition foliaire des arbres (voir figure 6 ainsi que des exemples d'autres études dans Thimonier et al 2010). L'absence de relation entre dépôts et concentrations foliaires chez les feuillus du réseau IAP n'est cependant pas en faveur de cette hypothèse (Braun et al 2010). Smidt (2007), en Autriche, a montré une diminution à la fois des dépôts et des concentrations foliaires en l'espace de dix ans, mais ces tendances n'étaient pas corrélées entre elles à l'échelle du site. La relation entre dépôts et concentrations foliaires n'est pas simple, comme le montre l'absence de corrélation pour les conifères une fois l'influence de l'altitude prise en compte. Cet effet de l'altitude sur les concentrations foliaires pourrait s'expliquer par des effets de photo-oxydation ou de températures plus basses (Polle et al 1999).

Une deuxième tendance se dessine pour la composition du feuillage: une réduction de la concentration en phosphore. Cette diminution est marquée pour les peuplements du réseau IAP, elle est esquissée pour les sites LWF, mais elle n'est pas observable à Alptal. Au sein du réseau IAP, elle est associée à une augmentation du rapport N/P vers des valeurs indiquant des proportions relatives défavorables en termes de nutrition. Un déclin des concen- trations en phosphore a été observé dans plusieurs autres études (Duquesnay et al 2000, Jonard et al 2009). De même, Khanna et al (2007) documentent une diminution des concentrations en phosphore chez l'épicéa et le pin de 1995 à 2005 en Allemagne (pas chez le hêtre cependant). Ces changements du statut nutritif concernant le phosphore pourraient être liés à des dépôts azotés élevés, via par exemple l'augmentation de la demande en cet élément à la suite de l'augmentation de la disponibilité en azote, de la réduction des mycorhizes par l'azote (Wallenda \& Kottke 1998) ou via l'acidification du sol. La diminution des concentrations foliaires en magnésium (résultats non montrés), observée sur les sites IAP chez le hêtre (pas sur les sites LWF) ainsi que d'autres sites en Europe (Duquesnay et al 2000) pourrait avoir la même origine. D'autres facteurs comme des années de forte fructification pourraient également contribuer à des concentrations en P plus basses à la suite de processus de translocation entre feuillage et fruits (Jonard et al 2009).

En conclusion, les dépôts d'azote tendent à diminuer au cours de ces dernières années, mais ils restent élevés au Tessin, sur le Plateau et sur les contreforts nord des Alpes. L'intensité du lessivage indique que certains sites sont saturés en azote. Nous n'observons pas de diminution du lessivage sur les sites où les dépôts ont diminué. Les concentrations foliaires en azote tendent à diminuer, mais elles restent dans la gamme de valeurs satisfaisantes, sauf pour certains peuplements de conifères. Le phosphore tend également à diminuer, à des niveaux indiquant une carence en cet élément. Certaines tendances détectées sur les sites des réseaux IAP, LWF et d'Alptal concordent. D'autres sont parfois visibles sur certains sites ou réseaux seulement, ce qui peut s'expliquer par la durée des périodes d'étude, par le nombre de sites, ou par les spécificités locales des sites étudiés, autant de facteurs qui diffèrent selon le réseau de recherche. Bien que la situation à l'égard de l'azote ne présente pas de risque général immédiat pour les écosystèmes forestiers, elle reste préoccupante et requiert la poursuite des observations sur le long terme.

Soumis: 12 décembre 2011, accepté (avec comité de lecture): 27 juillet 2012

\section{Remerciements}

Nos remerciements vont aux très nombreuses personnes qui, depuis plus de 15-25 ans, ont participé aux prélèvements et relevés sur les sites, préparé et analysé les échantillons, assuré l'archivage des données et participé à leur évaluation. Nous remercions également les cantons et l'OFEV pour leur soutien financier. 


\section{Références}

ABER J ET AL (1998) Nitrogen saturation in temperate forest ecosystems - Hypotheses revisited. BioSci 48: 921-934.

BOBBINK R, HETTELINGH J, EDITORS (2011) Review and revision of empirical critical loads and dose-response relationships. www.rivm.nl/bibliotheek/rapporten/680359002.pdf (28.7.2012)

BORKEN W, MATZNER E (2004) Nitrate leaching in forest soils: an analysis of long-term monitoring sites in Germany. J Plant Nutr Soil Sci 167: 277-283.

BRAEKKE FH, SALIH N (2002) Reliability of foliar analyses of Norway spruce stands in a Nordic gradient. Silva Fenn 36: 489504.

BRAUN-BLANQUET J (1964) Pflanzensoziologie. Grundzüge der Vegetationskunde. Wien: Springer, 3 ed. 865 p.

BRAUN S, FLÜCKIGER W (2012) Bodenversauerung in den Beobachtungsflächen des Interkantonalen Walddauerbeobachtungsprogramms. Schweiz Z Forstwes 163: 374-382. doi: 10.3188/szf.2012.0374

BRAUN S, RIHM B, FLÜCKIGER W (2012) Stickstoffeinträge in den Schweizer Wald: Ausmass und Auswirkungen. Schweiz Z Forstwes 163: 355-362. doi: 10.3188/szf.2012.0355

BRAUN S, THOMAS VFD, QUIRING R, FLÜCKIGER W (2010) Does nitrogen deposition increase forest production? The role of phosphorus. Environ Pollut 158: 2043-2052.

CFHA (2005) Les polluants atmosphériques azotés en Suisse. Rapport de la Commission fédérale de I'hygiène de I'air (CFHA). Berne: Office fédéral de l'environnement, des forêts et du paysage, Cahier de l'environnement. $174 \mathrm{p}$.

DE VRIES W, VAN DER SALM C, REINDS GJ, ERISMAN JW (2007) Element fluxes through European forest ecosystems and their relationships with stand and site characteristics. Environ Pollut 148: 501-513.

DE VRIES W ET AL (2003) Intensive monitoring of forest ecosystems in Europe: 1. Objectives, set-up and evaluation strategy. For Ecol Manage 174: 77-95.

DIEKMANN M, BRUNET J, RÜHLING A, FALKENGREN-GRERUP U (1999) Effects of nitrogen deposition: Results of a temporalspatial analysis of deciduous forests in South Sweden. Plant Biol 1: 471-481.

DISE NB, ROTHWELL JJ, GAUCI V, VAN DER SALM C, DE VRIES W (2009) Predicting dissolved inorganic nitrogen leaching in European forests using two independent databases. Sci Total Environ 407: 1798-1808.

DUQUESNAY A, DUPOUEY JL, CLÉMENT A, ULRICH E, LE TACON F (2000) Spatial and temporal variability of foliar mineral concentration in beech (Fagus sylvatica) stands in northeastern France. Tree Physiol 20: 13-22.

ELLENBERG H, WEBER HE, DÜLL R, WIRTH V, WERNER W (1992) Zeigerwerte der Pflanzen in Mitteleuropa. Scr Geobot 18: $1-262$.

ERISMAN JW, DE VRIES W (2000) Nitrogen deposition and effects on European forests. Environ Rev 8: 65-93.

FLÜCKIGER W, BRAUN S (1998) Nitrogen deposition in Swiss forests and its possible relevance for leaf nutrient status, parasite attacks and soil acidification. Environ Pollut 102: 69-76.

FLÜCKIGER W, BRAUN S (2003) Critical limits for nutrient concentrations and ratios for forest trees - a comment. Berne: Swiss Agency Environment Forests Landscape, Environmental Documentation 164. 280 p.

FLÜCKIGER W, BRAUN S, MAINIERO R, SCHÜTZ K, THOMAS V (2011) Auswirkung erhöhter Stickstoffbelastung auf die Stabilität des Waldes. Schönenbuch: Institut angewandte Pflanzenbiologie. $87 \mathrm{p}$.

GRAF PANNATIER E, DOBBERTIN M, SCHMITT M, THIMONIER A, WALDNER P (2009) L'eau des sols forestiers: un milieu sensible aux changements. Langzeitforschung für eine nachhaltige Waldnutzung. Birmensdorf: Eidgenöss Forsch.anstalt WSL, Forum Wissen. pp. 21-30.
GRAF PANNATIER E, THIMONIER A, SCHMITT M, WALDNER P, WALTHERT L (2012) Impacts des dépôts atmosphériques acides sur l'eau des sols forestiers. Schweiz Z Forstwes 163: 363-373. doi: 10.3188/szf.2012.0363

GRAF PANNATIER E, THIMONIER A, SCHMITT M, WALTHERT L, WALDNER P (2011) A decade of monitoring at Swiss Longterm Forest Ecosystem Research (LWF) sites: can we observe trends in atmospheric acid deposition and in soil solution acidity? Environ Monit Assess 174: 3-30.

GRAF PANNATIER E, WALTHERT L, BLASER P (2004) Solution chemistry in acid forest soils: Are the BC:Al ratios as critical as expected in Switzerland? J Plant Nutr Soil Sci 167: 160-168.

HELDSTAB J, REUTIMANN J, BIEDERMANN R, LEU D (2010) Stickstoffflüsse in der Schweiz. Stoffflussanalyse für das Jahr 2005. Bern: Bundesamt Umwelt. 128 p.

INNES JL (1995) Theoretical and practical criteria for the selection of ecosystem monitoring plots in Swiss forests. Environ Monit Assess 36: 271-294.

JANSSON PE, KARLBERG L (2004) Coupled heat and mass transfer model for soil-plant-atmosphere systems. Stockholm: Roy Inst Techn, Dept Civil Environm Engin. 435 p.

JONARD M, ANDRÉ F, DAMBRINE E, PONETTE Q, ULRICH E (2009) Temporal trends in the foliar nutritional status of the French, Walloon and Luxembourg broad-leaved plots of forest monitoring. Ann For Sci 66: 412.

KHANNA P ET AL (2007) Assessment of changes in the phosphorus status of forest ecosystems in Germany - Literature review and analysis of existing data. Freiburg: Univ Freiburg, Institute Silviculture. $85 \mathrm{p}$.

KUHN N, AMIET R, HUFSCHMID N (1987) Veränderungen in der Waldvegetation der Schweiz infolge Nährstoffanreicherungen aus der Atmosphäre. Allg Forst- Jagdztg 158: 77-84.

LANDOLT E (1977) Ökologische Zeigerwerte zur Schweizer Flora. Veröff Geobot Inst Eidgenöss Tech Hochsch Stift Rübel Zür 64. $208 \mathrm{p}$.

MELLERT KH, PRIETZEL J, STRAUSSBERGER R, REHFUESS KE (2004) Long-term nutritional trends of conifer stands in Europe: results from the RECOGNITION project. Eur J For Res 123: 305-319.

MOHR K ET AL (2005) Bestimmung von Ammoniak-Einträgen aus der Luft und deren Wirkung auf Waldökosysteme (ANSWER-Projekt). http://literatur.vti.bund.de/digbib_extern/bitv/ zi036910.pdf (31.7.2012)

OFEV (2011) NABEL - La pollution de l'air en 2010. Résultats du Réseau national d'observation des polluants atmosphériques (NABEL). Berne: Office fédéral de I'environnement, Etat de I'environnement. $126 \mathrm{p}$.

POLLE A ET AL (1999) Growth and protection against oxidative stress in young clones and mature spruce trees (Picea abies L.) at high altitudes. Oecologia 121: 149-156.

RIHM B (1994) Critical loads of acidity for forest soils and alpine lakes. Steady state mass balance method. Berne: Federal Office Environment Forests Landscape, Environmental Series Air. $68 \mathrm{p}$.

SCHLEPPI P, MORIER I, PROVIDOLI I (2006A) Saturation en azote et lessivage de nitrate dans une forêt subalpine soumise pendant 10 ans à des dépôts d'azote expérimentalement accrus. Bull Soc Suisse Pédol 29: 15-20.

SCHLEPPI P, MULLER N, EDWARDS PJ, BUCHER JB (1999) Three years of increased nitrogen deposition do not affect the vegetation of a montane forest ecosystem. Phyton 39: 197-204.

SCHLEPPI P ET AL (1998) Nitrogen budgets of two small experimental forested catchments at Alptal, Switzerland. For Ecol Manage 101: 177-185.

SCHLEPPI P, WALDNER PA, FRITSCHI B (2006B) Accuracy and precision of different sampling strategies and flux integration methods for runoff water: comparisons based on measurements of the electrical conductivity. Hydrol Process 20: 395-410. 
SCHULLA J, JASPER K (2007) Model description WaSiM-ETH. http://wasim.ch/downloads/doku/wasim/wasim_2007_en.pdf (31.7.2012)

SLOOTWEG J, POSCH M, HETTELINGH J-P (EDS.) (2007) Critical loads of nitrogen and dynamic modelling. CCE progress report 2007. www.pbl.nl/sites/default/files/cms/publicaties/ 500090001.pdf (31.7.2012)

SMIDT S (2007) 10 Jahre Depositionsmessung im Rahmen des europäischen Waldschadensmonitorings. Ergebnisse 19962005, BFW-Berichte. Wien: Bundesforschungs-Ausbildungszentrum Wald Naturgefahren Landschaft. 79 p.

SOLBERG S ET AL (2009) Analyses of the impact of changes in atmospheric deposition and climate on forest growth in European monitoring plots: A stand growth approach. For Ecol Manage 258: 1735-1750.

STEFAN K, FÜRST A, HACKER R, BARTELS U (1997) Forest foliar condition in Europe - Results of large-scale foliar chemistry surveys (survey 1995 and data from previous years). http:// bfw.ac.at/600/ffcc/report_forest_condition.zip (31.7.2012)

THIMONIER A (1998) Variabilité spatio-temporelle des dépôts atmosphériques d'éléments minéraux sous hêtraie. Schweiz Z Forstwes 149: 585-614.

\section{Dépôts atmosphériques azotés et leurs effets en forêt: un bilan des sites d'obser- vation à long terme}

Les émissions de polluants azotés ont atteint un pic dans le milieu des années 1980 et ont diminué depuis, mais elles restent à un niveau élevé. L'inquiétude suscitée par d'éventuels effets de la pollution sur la vitalité des forêts a motivé la mise en place de sites d'observation à long terme. De tels sites ont ainsi été établis en Suisse entre 1984 et 1995 au sein de trois réseaux (IAP, LWF et NITREX). Notre objectif est ici de caractériser la disponibilité en azote des sites étudiés et son évolution en l'espace de 15 et 25 ans. Les apports atmosphériques d'azote ont été obtenus pour chaque site par un modèle (IAP) ou à partir de l'échantillonnage des précipitations sous forêt (LWF et NITREX). Les indicateurs de la disponibilité en azote d'un site comprennent le lessivage de nitrate en dessous de la zone racinaire (43 sites), les concentrations foliaires des arbres en azote et en autres nutriments (>100 sites) et la composition floristique de la végétation du sous-bois (59 sites). Les dépôts atmosphériques d'azote ont diminué significativement sur 8 sites sur 12 (LWF) au cours des 15 dernières années. Cependant, sauf dans les Alpes, ils se situent dans la gamme de charges critiques empiriques pour l'azote, voire excèdent ces charges critiques, qui correspondent aux dépôts au-dessus desquels des changements négatifs peuvent affecter l'écosystème. Le lessivage de nitrate peut être localement élevé. Il dépend de l'amplitude des dépôts, mais aussi d'autres facteurs comme les propriétés du sol. Les concentrations foliaires des arbres en azote et phosphore ont eu tendance à diminuer au cours des 15-25 ans. La comparaison de relevés de végétation, effectués d'abord en 1984, puis répétés en 2003/2004, indique une augmentation du caractère nitrophile de la végétation (IAP). Sur un plus petit nombre de sites (LWF), sur une période plus courte et plus récente (entre 1994 et 2011), nous n'observons par contre pas de changements significatifs.
THIMONIER A, DUPOUEY JL, TIMBAL J (1992) Floristic changes in the herb-layer vegetation of a deciduous forest in the Lorraine Plain under the influence of atmospheric deposition. For Ecol Manage 55: 149-167.

THIMONIER A ET AL (2010) Does exceeding the critical loads for nitrogen alter nitrate leaching, the nutrient status of trees and their crown condition at Swiss long-term forest ecosystem research (LWF) sites? Eur J For Res 129: 443-461.

THIMONIER A, KULL P, KELLER W, MOSER B, WOHLGEMUTH T (2011) Ground vegetation monitoring in Swiss forests: comparison of survey methods and implications for trend assessments. Environ Monit Assess 174: 47-63.

THIMONIER A, SCHMITT M, WALDNER P, RIHM B (2005) Atmospheric deposition on Swiss Long-term Forest Ecosystem Research (LWF) plots. Environ Monit Assess 104: 81-118.

WALLENDA T, KOTTKE I (1998) Nitrogen deposition and ectomycorrhizas. New Phytol 139: 169-187.

WRIGHT RF, RASMUSSEN L (1998) Introduction to the NITREX and EXMAN projects. For Ecol Manage 101: 1-7.

\section{Stickstoffdeposition und deren Auswirkungen auf den Wald - eine Bilanz anhand von langfristigen Beobachtungsflächen}

Die Stickstoffemissionen erreichten Mitte der 1980er-Jahre einen Höchststand. Seither nehmen sie kontinuierlich ab, bleiben aber auf hohem Niveau. Aufgrund der Befürchtung, dass die Vitalität der Wälder durch die Luftverschmutzung beeinträchtigt würde, wurden in der Schweiz in den Jahren 1984 bis 1995 im Rahmen der drei Netzwerke IAP, LWF und NITREX langfristige Beobachtungsflächen eingerichtet, auf denen während der letzten 10 bis 25 Jahre die Stickstoffverfügbarkeit und deren Entwicklung gemessen wurden. Der Stickstoffeintrag wurde für jeden Standort entweder modelliert (IAP) oder aufgrund von Bestandesniederschlagsmessungen (LWF, NITREX) geschätzt. Als Indikatoren für die Stickstoffverfügbarkeit einer Fläche dienten die Nitratauswaschung aus dem Wurzelhorizont (43 Flächen), die Stickstoff- und Nährstoffkonzentration in den Blättern und Nadeln (>100 Flächen) sowie die Zusammensetzung der Bodenvegetation (59 Flächen). Auf acht von zwölf Beobachtungsflächen (LWF) ist der Stickstoffeintrag während der vergangenen 15 Jahre signifikant zurückgegangen. Abgesehen von den alpinen Standorten liegen die Stickstoffeinträge jedoch immer noch im Bereich der Critical Loads oder überschreiten diese. Nach heutigem Wissensstand kann das Ökosystem Wald bei Stickstoffeinträgen über den Critical Loads nachhaltig geschädigt werden. Lokal wurden hohe Nitratauswaschungen aus dem Wurzelhorizont gemessen, was von der Stickstoffdeposition und weiteren Faktoren wie beispielsweise den Bodeneigenschaften abhängt. Der Stickstoff- und Phosphorgehalt in den Blättern und Nadeln hat während den letzten 15 bis 25 Jahren tendenziell abgenommen. Der Vergleich der Vegetationsaufnahmen im Jahr 1984 mit jenen in den Jahren 2003 und 2004 (IAP) zeigt eine Begünstigung der stickstoffliebenden Arten. Ein neuerer Vergleich zwischen 1994 und 2011 auf einer kleineren Anzahl Flächen (LWF) zeigt hingegen keine signifikante Änderung in der Zusammensetzung der Bodenvegetation. 